\title{
ON A FORMULA OF A. C. DIXON
}

T. S. NANJUNDIAH

1. A well known formula of A. C. Dixon [1], which is equivalent to the functional identity

$$
\begin{aligned}
\left(1+\frac{x}{(1 !)^{3}}+\frac{x^{2}}{(2 !)^{3}}+\cdots\right) & \left(1-\frac{x}{(1 !)^{3}}+\frac{x^{2}}{(2 !)^{3}}-\cdots\right) \\
= & 1-\frac{3 !}{(1 ! 2 !)^{3}} x^{2}+\frac{6 !}{(2 ! 4 !)^{3}} x^{4}-\cdots
\end{aligned}
$$

due to Ramanujan [2], reads:

$$
\sum_{r=0}^{2 n}(-1)^{r}\left(\begin{array}{c}
2 n \\
r
\end{array}\right)^{3}=(-1)^{n} \frac{(3 n) !}{(n !)^{3}}, \quad n=0,1,2, \cdots .
$$

In their proper setting, the formulae (1) and (2) belong to the theory of hypergeometric series. We however present below a very simple and elementary approach to (2) leading to some generalizations from which this formula appears in a new light. As is well known, the first elementary proof of (2) is due to H. W. Richmond [5]. For two other elementary proofs of more recent origin, we refer to the papers ${ }^{1}$ [3] and [6].

2. We can clearly set up the identity

$$
\sum_{r=0}^{n}\left(\begin{array}{l}
n \\
r
\end{array}\right)^{p} \alpha^{n-r} \beta^{r}=\sum_{r=0}^{[n / 2]} C_{n, r}^{(p)}\left(\begin{array}{c}
n-r \\
r
\end{array}\right)(\alpha+\beta)^{n-2 r}(\alpha \beta)^{r},
$$

where the coefficients $C_{n, r}^{(p)}$ are successively determined by the equations

$$
\left(\begin{array}{l}
n \\
k
\end{array}\right)^{p}=\sum_{r=0}^{k} C_{n, r}^{(p)}\left(\begin{array}{c}
n-r \\
r
\end{array}\right)\left(\begin{array}{c}
n-2 r \\
k-r
\end{array}\right), \quad k=0,1, \cdots,[n / 2],
$$

expressing the equality of the coefficients of $\alpha^{n-k} \beta^{k}$ on both sides of (3). In fact, (3) and (4) are completely equivalent.

Observe that (4) may be rewritten in the form

$$
\left(\begin{array}{l}
n \\
k
\end{array}\right)^{p}=\sum_{r=0}^{k} C_{n, r}^{(p)}\left(\begin{array}{c}
k \\
r
\end{array}\right)\left(\begin{array}{c}
n-r \\
k
\end{array}\right) \text {. }
$$

Received by the editors July 25, 1957.

1 These were not available to the author, except for their report in the Mathematical Reviews. 
Using Vandermonde's identity, we can express the right member in (5) as a repeated sum obtaining

$$
\left(\begin{array}{l}
n \\
k
\end{array}\right)^{p}=\sum_{r=0}^{k} C_{n, r}^{(p)}\left(\begin{array}{c}
k \\
r
\end{array}\right) \sum_{s=r}^{k}\left(\begin{array}{c}
n-k \\
s
\end{array}\right)\left(\begin{array}{c}
k-r \\
k-s
\end{array}\right) .
$$

Changing the order of summation on the right and observing that

$$
\left(\begin{array}{l}
k \\
r
\end{array}\right)\left(\begin{array}{l}
k-r \\
k-s
\end{array}\right)=\left(\begin{array}{l}
k \\
s
\end{array}\right)\left(\begin{array}{l}
s \\
r
\end{array}\right)
$$

we have

$$
\left(\begin{array}{l}
n \\
k
\end{array}\right)^{p}=\sum_{s=0}^{k}\left(\begin{array}{c}
k \\
s
\end{array}\right)\left(\begin{array}{c}
n-k \\
s
\end{array}\right) \sum_{r=0}^{s} C_{n, r}^{(p)}\left(\begin{array}{l}
s \\
r
\end{array}\right)
$$

On multiplying by

$$
\left(\begin{array}{l}
n \\
k
\end{array}\right)
$$

and observing that

$$
\left(\begin{array}{c}
n \\
k
\end{array}\right)\left(\begin{array}{c}
n-k \\
s
\end{array}\right)=\left(\begin{array}{c}
n \\
s
\end{array}\right)\left(\begin{array}{c}
n-s \\
k
\end{array}\right)
$$

we have

$$
\left(\begin{array}{l}
n \\
k
\end{array}\right)^{p+1}=\sum_{s=0}^{k}\left(\begin{array}{l}
k \\
s
\end{array}\right)\left(\begin{array}{c}
n-s \\
k
\end{array}\right) \cdot\left(\begin{array}{l}
n \\
s
\end{array}\right) \sum_{r=0}^{s}\left(\begin{array}{l}
s \\
r
\end{array}\right) C_{n, r \cdot}^{(p)}
$$

Comparison of (5) and (6) establishes for the $C_{n, r}^{(p)}$ the following recursion with respect to $p$ :

$$
C_{n, s}^{(p+1)}=\left(\begin{array}{c}
n \\
s
\end{array}\right) \sum_{r=0}^{s}\left(\begin{array}{l}
s \\
r
\end{array}\right) C_{n, r}^{(p)}, \quad 0 \leqq s \leqq[n / 2] .
$$

Now for $p=1,(3)$ gives (binomial identity !):

$$
C_{n, r}^{(1)}=\left(\begin{array}{l}
0 \\
r
\end{array}\right)
$$

whence (7) yields, by iteration, the following explicit representation for the $C_{n, r}^{(p)}(p=1,2,3, \cdots)$ :

(9) $\quad C_{n, r}^{(p)}=\sum_{0 \leqq r_{1} \leq \cdots \leq r_{p} \leqq r}\left(\begin{array}{l}0 \\ r_{1}\end{array}\right)\left(\begin{array}{l}r_{2} \\ r_{1}\end{array}\right) \cdots\left(\begin{array}{c}r_{p} \\ r_{p-1}\end{array}\right)\left(\begin{array}{c}r_{p} \\ r\end{array}\right)\left(\begin{array}{c}n \\ r_{1}\end{array}\right) \cdots\left(\begin{array}{l}n \\ r_{p}\end{array}\right)$.

In particular, we have 


$$
C_{n, r}^{(2)}=\left(\begin{array}{c}
n \\
r
\end{array}\right), \quad C_{n, r}^{(3)}=\left(\begin{array}{c}
n+r \\
r
\end{array}\right)\left(\begin{array}{c}
n \\
r
\end{array}\right) .
$$

In deriving the latter formula in (10), Vandermonde's identity has been used. Taking $p=3, \alpha=1, \beta=-1$ and replacing $n$ by $2 n$ in (3), we obtain (2). The case $p=3$ of (3) has been derived by P. A. MacMahon [4] by another procedure which is not altogether elementary.

3. If, in (7), which evidently holds for arbitrary $p$, we write $-(p+1)$ for $p$ and define $d_{n, r}^{(p)}$ with

$$
C_{n, r}^{(-p)}=(-1)^{r} d_{n, r}^{(p)}
$$

it readily takes the form

$$
\left(\begin{array}{l}
n \\
s
\end{array}\right)^{-1} d_{n, s}^{(p)}=\Delta^{s} d_{n, 0}^{(p+1)}
$$

Here we have used the notation of finite differences with $\Delta$ operating on the index $s$ in $d_{n, s}^{(p)}$. Now

$$
d_{n, s}^{(p+1)}=E^{s} d_{n, 0}^{(p+1)}=(1+\Delta)^{s} d_{n, 0}^{(p+1)}=\sum_{r=0}^{s}\left(\begin{array}{c}
s \\
r
\end{array}\right) \Delta^{r} d_{n, 0}^{(p+1)} .
$$

Herewith we secure the following recursion for the $d_{n, r}^{(p)}$ with respect to $p$ :

$$
d_{n, s}^{(p+1)}=\sum_{r=0}^{s}\left(\begin{array}{l}
s \\
r
\end{array}\right)\left(\begin{array}{l}
n \\
r
\end{array}\right)^{-1} d_{n, r}^{(p)}, \quad 0 \leqq s \leqq[n / 2] .
$$

We have, according to (8) and the definition (11),

$$
d_{n, r}^{(-1)}=\left(\begin{array}{l}
0 \\
r
\end{array}\right)
$$

whence (12) yields, by iteration, the following explicit representation for the $d_{n, r}^{(p)}(p=0,1, \cdots)$ :

$$
d_{n, r}^{(p)}=
$$

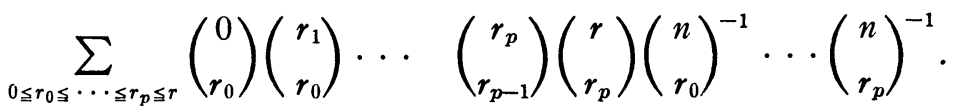

In particular, we have 


$$
d_{n, r}^{(0)}=1, \quad d_{n, r}^{(1)}=\left(\begin{array}{c}
n+1 \\
r
\end{array}\right)\left(\begin{array}{c}
n \\
r
\end{array}\right)^{-1} .
$$

In deriving the latter formula in (15), we have made use of the relations

$$
\left(\begin{array}{l}
n \\
r
\end{array}\right)\left(\begin{array}{l}
r \\
s
\end{array}\right)=\left(\begin{array}{c}
n \\
s
\end{array}\right)\left(\begin{array}{c}
n-s \\
r-s
\end{array}\right), \quad\left(\begin{array}{c}
n+1 \\
r
\end{array}\right)=\sum_{s=0}^{r}\left(\begin{array}{c}
n-s \\
r-s
\end{array}\right), \quad s \leqq r .
$$

We thus have the interesting identity:

$$
\begin{aligned}
\sum_{r=0}^{n}\left(\begin{array}{l}
n \\
r
\end{array}\right)^{-1} \alpha^{n-r} \beta^{r} & \\
& =\sum_{r=0}^{[n / 2]}(-1)^{r}\left(\begin{array}{c}
n+1 \\
r
\end{array}\right)\left(\begin{array}{c}
n \\
r
\end{array}\right)^{-1}\left(\begin{array}{c}
n-r \\
r
\end{array}\right)(\alpha+\beta)^{n-2 r}(\alpha \beta)^{r} .
\end{aligned}
$$

This gives, as a special case with $\alpha=1, \beta=-1$ and $2 n$ in place of $n$, the neat formula

$$
\sum_{r=0}^{2 n}(-1)^{r} /\left(\begin{array}{c}
2 n \\
r
\end{array}\right)=(2 n+1) /(n+1), \quad n=0,1,2, \cdots .
$$

This is analogous to (2), but not so interesting. In fact it is a particular case of the more readily derivable formula

$$
\begin{aligned}
\sum_{r=0}^{n}(-1)^{r} /\left(\begin{array}{c}
m-1 \\
r
\end{array}\right)=\frac{m}{m+1}\left\{1+(-1)^{n} /\left(\begin{array}{c}
m \\
n+1
\end{array}\right)\right\}, \\
m>n \geqq 0 .
\end{aligned}
$$

For the proof of (18), we need only notice the relations

$$
m\left(\begin{array}{c}
m-1 \\
r
\end{array}\right)=(m-r)\left(\begin{array}{c}
m \\
r
\end{array}\right)=(r+1)\left(\begin{array}{c}
m \\
r+1
\end{array}\right), \quad 0 \leqq r \leqq n .
$$

\section{REFERENCES}

1. A. C. Dixon, On the sum of the cubes of the coefficients in a certain expansion by the binomial theorem, Messenger of Mathematics vol. 20 (1891) pp. 79-80.

2. G. H. Hardy, Ramanujan, Cambridge, 1940, p. 7.

3. Wilhelm Ljunggren, $A n$ elementary proof of a formula of $A$. C. Dixon, Norsk Mat. Tidsskr. vol. 29 (1947) pp. 35-38.

4. P. A. MacMahon, The sums of powers of the binomial coefficients, Quart. J. Math. vol. 33 (1902) pp. 274-288.

5. H. W. Richmond, The sum of the cubes of the coefficients in $(1-x)^{2 n}$, Messenger of Mathematics vol. 21 (1892) pp. 77-78.

6. Tor B. Staver, On summation of powers of binomial coefficients, Norsk. Mat. Tidsskr. vol. 29 (1947) pp. 97-103.

Central College, Bangalore, India 